\title{
Prednisone for the treatment of withdrawal headache in patients with medication overuse headache: A randomized, double-blind, placebo-controlled study
}

33(3) 202-207

(C) International Headache Society 2012

Reprints and permissions:

sagepub.co.uk/journalsPermissions.nav DOI: $10.1177 / 0333102412462638$ cep.sagepub.com

(S)SAGE

\author{
Kasja Rabe', Lutz Pageler ${ }^{1,2}$, Charly Gaul ${ }^{1,3}$, Christian \\ Lampl $^{4}$, Torsten Kraya ${ }^{3}$, Stefanie Foerderreuther ${ }^{5}$, \\ Hans-Christoph Diener ${ }^{1}$ and Zaza Katsarava ${ }^{1,6}$
}

\begin{abstract}
Purpose: To investigate the efficacy of prednisone for treatment of withdrawal headache in patients with medication overuse headache $(\mathrm{MOH})$.

Patients and methods: In this prospective double-blind, placebo-controlled, parallel designed multicentre trial, 96 consecutive patients with $\mathrm{MOH}$ were randomized to withdrawal treatment with either $100 \mathrm{mg}$ prednisone or placebo over 5 days. Patients were enrolled if they met the International Headache Society criteria for $\mathrm{MOH}$ and were diagnosed with migraine or episodic tension-type headache as primary headache. Exclusion criteria comprised significant neurological or psychiatric disorders. Withdrawal symptoms, including headache severity and intake of rescue medication, were documented for 14 days after randomization.

Results: Patients treated with prednisone did not experience fewer hours of moderate or severe headache than patients receiving placebo. However, patients requested less rescue medication within the first 5 days.

Conclusions: During withdrawal in $\mathrm{MOH}$, prednisone reduces rescue medication without decreasing the severity and duration of withdrawal headache.
\end{abstract}

\section{Keywords}

Medication overuse headache, withdrawal headache, prednisone

Date received: 4 March 20I2; revised: 3I May 20I2; 10 August 20I2; accepted: 2 September 2012

\section{Introduction}

Medication overuse headache $(\mathrm{MOH})$ is a widespread disorder with a prevalence of about $1-2 \%$ in the general population (1). Medication overuse headache is usually diagnosed in patients with primary headache, such as migraine or tension-type headache, who develop daily or nearly daily headache on at least 15 days per month following overuse of acute headache medication (2). Treatment of choice for $\mathrm{MOH}$ is based on abrupt or gradual withdrawal of overused medication; however, this results in severe rebound headache and additional symptoms like nausea, arterial hypotension, tachycardia, sleep disturbances, anxiety, restlessness and nervousness in many patients (3). Treatment options for withdrawal headache are limited (4-6). Prednisone is suggested for treatment of withdrawal headache, but studies so far have provided mixed results (7-10).
In this multicentre, double-blind, randomized, placebocontrolled trial, we aimed to evaluate the efficacy of

\footnotetext{
'Department of Neurology and Headache Center, University DuisburgEssen, Germany

${ }^{2}$ Department of Neurology, Cologne City Hospitals, Germany

${ }^{3}$ Department of Neurology, Luther-University Halle-Wittenberg, Germany

${ }^{4}$ Department of Neurology and Pain Medicine, Upper Austrian Headache Centre, Konventhospital Barmherzige Brueder, Austria

${ }^{5}$ Department of Neurology, Ludwig-Maximilians-University, Germany

${ }^{6}$ Department of Neurology, Evangelisches Krankenhaus Unna, Germany
}

Corresponding author:

Kasja Rabe, Department of Neurology, University of Essen, Hufelandstr. 55, 45122 Essen, Germany.

Email: kasja.rabe@uk-essen.de 
prednisone in the treatment of withdrawal headache in patients with $\mathrm{MOH}$.

\section{Methods and patients}

Patients were enrolled if they met the International Headache Society criteria for MOH (11) and were diagnosed with migraine or episodic tension-type headache as primary headache. Exclusion criteria comprised significant neurological, psychiatric or other disorders and contraindications for treatment with prednisone according to the German SPC. Pregnant and breastfeeding women were excluded. Patients gave written consent after oral and written information. The local ethics committees of all participating centres approved the study. Patients were recruited from and included at headache clinics of the neurological departments of the university hospital of Essen, Luther-University HalleWittenberg, Ludwig-Maximilians-University Munich in Germany and in the Department of Neurology and Pain Medicine, Upper Austrian Headache Centre, of Konventhospital Barmherzige Brueder, Linz in Austria. Patient enrolment was relatively low, possibly because no allowance was paid for enrolment and participation in the trial.

\section{Study design}

The study was designed as a double-blind, placebocontrolled, randomized, parallel multicentre trial. A sample size of 96 patients (48 placebo, 48 verum) was allocated based on biometric calculations. A reduction of $20 \%$ of hours with moderate or severe headache during 5 days of withdrawal was expected. Interim analyses were planned per protocol after inclusion of 32 and 64 patients to allow premature assessment of significant differences. No significant differences were found.

Due to changing routine in the treatment of $\mathrm{MOH}$ patients, the protocol was adapted after inclusion of nine subjects. Patients could undergo withdrawal therapy not only as inpatients (as initially planned), but also on an outpatient basis.

Placebo or prednisone $100 \mathrm{mg}$, each in two tablets, was given once daily during the first 5 days of withdrawal. In addition, all patients were treated with calcium, potassium and $300 \mathrm{mg}$ ranitidin per day. The medication was blinded and randomized in a block size of four by standardized computer-based randomization software and delivered by the pharmacy of the University Hospital of Essen. Two of four consecutive patients received placebo and two received verum. Only the pharmacist was not blinded to treatment.

Patients were allowed to take rescue medication in case of unbearable headache, but were instructed to limit it as far as possible. The treating physician assessed dose and type of rescue medication. The frequency of intake of individual rescue medication was assessed. Prophylactic medication could be started during withdrawal. Severity of headache was documented as an average value every hour in a diary using a four-point scale $(0=$ none, $1=$ mild, $2=$ moderate, $3=$ severe headache) for 14 days. Documentation was interrupted during sleep. Patients were provided with stamped, addressed envelopes and asked to return diaries after completion. Patients were not asked to return to the clinic after completion of the headache diary. The primary endpoint was the number of hours with severe or moderate headache within the first 3 days of withdrawal. Secondary endpoints were the number of hours with severe or moderate headache within the first 5 and 14 days of withdrawal, and rescue medication used in each group. In addition, analogous to Bøe et al. (9), a mean headache index (MHI) was calculated based on the mean intensity of headache during withdrawal and days of withdrawal. $\mathrm{MHI}=(\mathrm{a} * 1+\mathrm{b} * 2+\mathrm{c} * 3) /$ number of hours recorded, where 'a' refers to number of days with mild headache, ' $b$ ' refers to number of days with moderate headache and ' $c$ ' refers to number of days with severe headache.

\section{Statistical analysis}

We compared mean values (age; period of primary headache; headache during the last 3 months; hours with severe or moderate headache within 3,5 and 14 days; hours with mild, moderate or severe headache within 3,5 or 14 days; rescue medication (intake frequency) within 5 and 14 days) between groups (prednisone; placebo) using independent-samples $t$-test and ratios (sex; primary headache; management) between groups using the Mann-Whitney-U-test. We performed ANOVA for repeated measurements to compare moderate and severe headache per day between groups (within-subjects variable TIME: hours with headache on days $1-3,3-5$ and 6-14); between-subjects variable GROUP: placebo, prednisone). All statistical calculations were performed using the software PASW statistics version 18 for Windows. The level of significance was set at 0.05 . Bonferroni correction was performed if appropriate.

\section{Results}

\section{Patient population}

Ninety-six patients (48 patients in each group) aged 18-68 years (mean (SD) 41 (12) years) were included between June 2004 and June 2009 in three centres in Germany (Neurological Departments of the University Hospitals of Essen, Halle (Saale) and Munich) and one 
centre in Austria (Department of Neurology and Pain Medicine, Upper Austrian Headache Centre, Konventhospital Barmherzige Brueder, Linz). Data for the primary endpoint were available for 78 patients (37 in the prednisone group and 41 in the placebo group). Missing data were due to incomplete or unreturned headache diaries. None of the patients was excluded from participation after randomization or did not receive medication. Patient groups available for analysis did not differ significantly concerning demographic and clinical characteristics (Table 1). The trial ended after inclusion of all planned patients.

\section{Influence of prednisone on severity of headache and rescue medication}

Primary and secondary endpoints are reported in Table 2. Numbers of hours with severe or moderate headache within the first 3 days (primary endpoint) and within the first 5 days (secondary endpoint) or 14 days of withdrawal did not differ significantly between groups. Patients in the prednisone group requested rescue medication less frequently than those in the placebo group, which was significant concerning the first 5 days of withdrawal ( $p=0.021,1.1$ vs. 2.3 doses), but not significant concerning the first 14 days of withdrawal ( $p=0.105,3.6$ vs. 6.4 doses). Additionally, we did not find statistically significant differences between groups in the number of hours with mild, moderate or severe headache within the first 3,5 or 14 days of withdrawal. Withdrawal headache decreased significantly after withdrawal without differences between the prednisone and placebo groups (number of hours with moderate or severe headache, days-effect (days 1-3, days 4 and 5, days 6-14: $p<0.001, F=43.458$; group effect: NS, ANOVA; Figure 1). Mean headache index within

Table I. Demographic and clinical characteristics of patients available for primary endpoint evaluation.

\begin{tabular}{|c|c|c|c|}
\hline & Prednisone & Placebo & $p$ \\
\hline Mean age (minimum; maximum) in years & $43.2(19 ; 68)$ & $40.0(18 ; 66)$ & 0.251 \\
\hline Sex: female; male & f: $34 ; m: 3$ & f: $32 ; m: 9$ & 0.093 \\
\hline Primary headache diagnosis & & & 0.833 \\
\hline Migraine & 30 & 34 & \\
\hline Tension-type headache & 7 & 7 & \\
\hline Management & & & 0.214 \\
\hline Inpatients & 29 & 26 & \\
\hline Outpatients & 8 & 15 & \\
\hline Mean duration of primary headache in years (minimum; maximum) & $26.1(1 ; 50)$ & $20.8(3 ; 50)$ & 0.066 \\
\hline $\begin{array}{l}\text { Mean number of headache days per month during the previous } 3 \\
\text { months (minimum; maximum) }\end{array}$ & $26.4(14 ; 30)$ & $25.4(12 ; 30)$ & 0.479 \\
\hline \multicolumn{4}{|l|}{ Patients using acute medication before withdrawal } \\
\hline Triptans & 19 & 26 & NS \\
\hline Analgesics & 22 & 26 & NS \\
\hline Combination analgesics & 9 & II & NS \\
\hline containing caffeine & 5 & 5 & NS \\
\hline containing opioids & I & 2 & NS \\
\hline Ergots & 4 & 0 & 0.031 \\
\hline Others & 2 & 1 & NS \\
\hline \multicolumn{4}{|l|}{ Patients taking preventive medication during withdrawal } \\
\hline None & 13 & 8 & NS \\
\hline At least one & 24 & 33 & NS \\
\hline Beta blockers & 10 & 8 & NS \\
\hline Flunarizine & 3 & 1 & NS \\
\hline Topiramate & 13 & 16 & NS \\
\hline Valproic acid & 5 & 4 & NS \\
\hline Amitriptyline, -oxide & 6 & 6 & NS \\
\hline Other antidepressants & 5 & 8 & NS \\
\hline Others (Tizanidine, Telmisartan, Lamotrigine) & 2 & 5 & NS \\
\hline
\end{tabular}


Table 2. Severity of headache, other withdrawal symptoms and intake frequency of rescue medication taken.

\begin{tabular}{|c|c|c|c|}
\hline & Prednisone & Placebo & $t$-test $(p)$ \\
\hline \multicolumn{4}{|l|}{ Primary endpoint } \\
\hline Hours with severe or moderate headache within the first 3 days & 20.9 & 18.2 & NS \\
\hline \multicolumn{4}{|l|}{ Secondary endpoints } \\
\hline Hours with severe or moderate headache within 5 days & 29.6 & 27.7 & NS \\
\hline Hours with severe or moderate headache within 14 days & 74.9 & 69.8 & NS \\
\hline Rescue medication (intake frequency) within 14 days & 3.6 & 6.4 & NS \\
\hline \multicolumn{4}{|l|}{ Further results } \\
\hline Rescue medication (intake frequency) within the first 5 days & I.I & 2.3 & 0.021 \\
\hline MHI within 3 days & 1.25 & 1.25 & NS \\
\hline MHI within 5 days & 0.80 & 0.85 & NS \\
\hline MHI within 14 days & 0.91 & 0.96 & NS \\
\hline $\begin{array}{l}\text { Further withdrawal symptoms (nausea, vomiting, photo-, phono-, osmophobia) } \\
\text { within } 5 \text { days }\end{array}$ & 39.2 & 27.8 & NS \\
\hline
\end{tabular}

MHI: mean headache index.

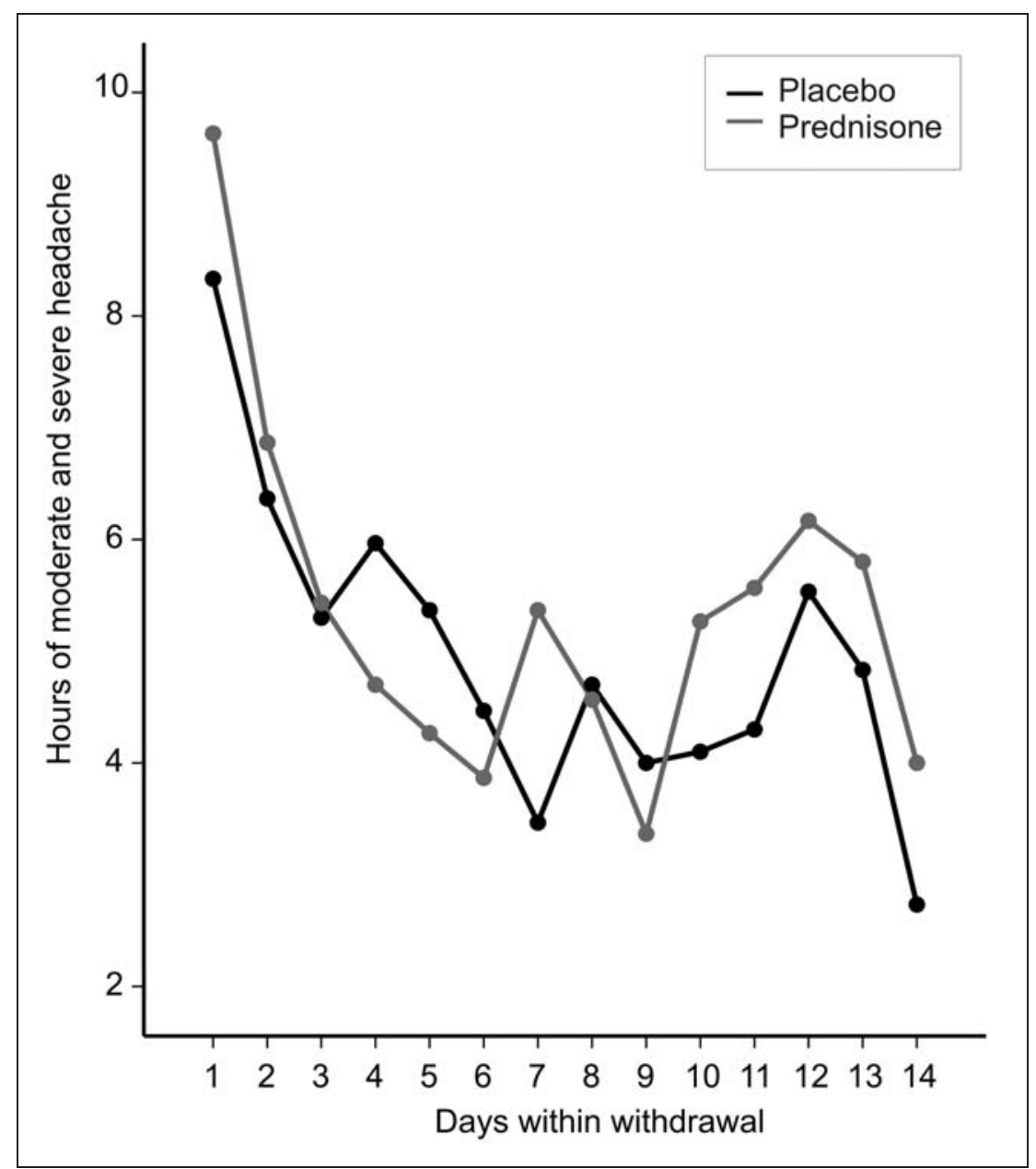

Figure I. Hours with moderate or severe headache decreased most within first 6-7 days of withdrawal. No statistically significant difference between groups was observed. 
3, 5 or 14 days of withdrawal did not differ significantly between groups. We did not find any differences comparing patients with migraine to those with episodic tension-type headache and comparing inpatient versus outpatient treatment. Reduction in headache was higher within the first 3 days in the group of patients using triptans than in patients using other analgesics, but differences were not statistically significantly (group effect; $p=0.493$; group by day effect; $p=0.081$ ). Prophylactic medication was taken by $51.3 \%$ of patients during withdrawal. We did not find significant differences in hours with moderate or severe headache during 3, 5 or 14 days of withdrawal between patients with or without preventive medication.

Treatment was well tolerated. One patient in the verum group developed diarrhoea for 1 day during treatment. A patient in the placebo group complained of dry mouth and dizziness. All symptoms resolved within 1 day. No other adverse events were reported. Because symptoms had to be reported by patients and were not inquired based on a list of possible adverse events, minor adverse events might have been disregarded. Within the first 5 days, $39.2 \%$ of patients in the prednisone group and $27.8 \%$ in the placebo group complained of additional expected withdrawal symptoms like nausea, vomiting, photo-, phono- and osmophobia (Table 2).

The following protocol deviations were identified: two patients did not fulfil inclusion criteria because they were older than 65 years. These patients were included in the analysis.

\section{Discussion}

Treatment with $100 \mathrm{mg}$ prednisone per day over 5 days in addition to rescue medication during withdrawal did not reduce hours of moderate or severe headache during withdrawal in patients with $\mathrm{MOH}$. Patients treated with placebo tended to need more rescue medication than patients taking prednisone.

It was suggested several years ago that prednisone might be effective in the treatment of withdrawal headache. The idea of using prednisone was attractive because a) it is effective in treating migrainous status (12) and b) in contrast to drugs usually used as rescue treatment such as intravenous aspirin, naproxen (6) or sumatriptan (5), prednisone does not bear the risk of supporting the vicious circle of medication overuse headache chronification. The mode of action is not known. Possible mechanisms are an anti-inflammatory effect and the inhibition of cyclo-oxygenase-II. Indeed, the first clinical observational study was promising, reporting improvement of headache following treatment with $60 \mathrm{mg}$ prednisone orally, but it did not compare results with placebo treatment (8). Our own small double-blind pilot study was the first randomized trial and results favoured prednisone over placebo (11). However, subsequent large placebo-controlled trials by Bøe et al. (9) and Krymchantowski et al. (7) were negative. In accordance with our current results, patients treated with placebo did not experience fewer hours of headache during withdrawal than patients treated with placebo. However, in their randomized trial, Krymchantowski et al. (7) found that patients treated with prednisone needed less acute pain medication (rescue drugs) during withdrawal than patients receiving naratriptan twice daily or no treatment. This observation is strengthened by our findings. Although headache frequency and severity are similar in both groups, patients treated with prednisone seem to have less need to take rescue drugs. We have to assume that prednisone can be used to reduce intake frequency of rescue medication during withdrawal. The following mechanisms have to be discussed. a) Prednisone has an analgesic effect, which is comparable to the effect of rescue medication. b) Prednisone reduces the longing for a rescue medication. With the assumption that prednisone does not support the circuit that headache leads to intake of pain medication, a theoretical advantage to conventional pain medication is imaginable. Because this effect is abolished within 14 days of withdrawal and patients use rescue medication with comparable frequency, we cannot give the recommendation to apply prednisone for 5 days in $\mathrm{MOH}$.

Combination analgesics were used infrequently in our patient group $(26 \%)$. This is within the range of other studies in Germany $(13,14)$. Withdrawal symptoms and prognosis are worse in patients using combination analgesics. More than half of the patients took triptans. Similar to previous reports $(14,15)$, we observed a trend that patients overusing triptans experienced a shorter and easier withdrawal. However, the differences were not significant.

The strength of the study is its design (multicentre, randomized, double-blind). We are aware of limitations. We have to acknowledge a drop-out rate of more than $10 \%$. We have considered several possibilities for improving compliance and quality of documentation. Admitting patients for 14 days to the hospital is not feasible at all centres and far from 'real life'. We called patients to remind them to return the headache diary. The task of documenting the intensity of headache every hour for 14 days required a high level of motivation and discipline and probably induced patients to drop out; but it did allow collection of valuable information about the course of withdrawal over the observation time. We did not assess hours of headache on days before withdrawal. Therefore, we cannot determine whether the headache intensity and number of hours with headache increased during withdrawal. 
Due to a very short observation period after withdrawal of 14 days, we cannot assess how many patients reverted to an episodic pattern after discontinuation of medication. Information about duration of $\mathrm{MOH}$ years and about earlier withdrawals could help to describe the patient population. Unfortunately, this information was not part of the study protocol and therefore is not available for most patients. Initiation of prophylactic treatment regimens during the withdrawal period according to clinical needs and the investigators' preferences might have biased the primary outcome data. There is still not enough evidence whether usage of prophylactic medication should be started on the day of withdrawal or after a few weeks, when the vicious circle of $\mathrm{MOH}$ has been broken. When this trial was started, it was common practice in Europe to first interrupt the overuse and begin with prophylactics later, if necessary. This was due to the awareness that prophylactics during overuse do not reduce headache frequency. Only recently it was shown that topiramate is effective even in patients overusing medication (16).

We conclude finally that our study suggests that prednisone is not effective in the treatment of withdrawal headache in patients with medication overuse headache, but can reduce intake of rescue medication during withdrawal.

\section{Clinical implications}

- To investigate the efficacy of prednisone for treatment of withdrawal headache in patients with medication overuse headache $(\mathrm{MOH})$.

- During withdrawal in $\mathrm{MOH}$, prednisone reduces rescue medication without decreasing the severity and duration of withdrawal headache.

\section{Funding}

Supported by the Federal Ministry of Education and Research (BMBF) as part of the German Headache Consortium (01EM0117).

\section{References}

1. Katsarava Z and Diener HC. Medication overuse headache in Germany. Cephalagia 2008; 28: 1221-1222.

2. Evers S and Marziniak M. Clinical features, pathophysiology, and treatment of medication-overuse headache. Lancet Neurol 2010; 9: 391-401.

3. Diener HC and Limmroth V. Medication-overuse headache: A worldwide problem. Lancet Neurol 2004; 3: 475-483.

4. Diener HC, Haab J, Peters C, et al. Subcutaneous sumatriptan in the treatment of headache during withdrawal from drug-induced headache. Headache 1990; 31: 205-209.

5. Mathew NT. Amelioration of ergotamine withdrawal with naproxen. Headache 1987; 27: 130-133.

6. Rossi P, Di Lorenzo C, Faroni J, et al. Advice alone vs. structured detoxification programmes for medication overuse headache: A prospective, randomized, open-label trial in transformed migraine patients with low medical needs. Cephalalgia 2006; 26: 1097-1105.

7. Krymchantowski AV and Barbosa JS. Prednisone as initial treatment of analgesic-induced daily headache. Cephalalgia 2000; 20: 107-113.

8. Krymchantowski AV and Moreira PF. Out-patient detoxification in chronic migraine: Comparison of strategies. Cephalalgia 2003; 23: 982-993.
9. Bøe MG, Mygland $\AA$ and Salvesen R. Prednisolone does not reduce withdrawal headache: A randomized, doubleblind study. Neurology 2007; 69: 26-31.

10. Pageler L, Katsarava Z, Diener HC, et al. Prednisone vs. placebo in withdrawal therapy following medication overuse headache. Cephalalgia 2008; 28: 152-156.

11. Headache Classification Committee of the International Headache Society. The International Classification of Headache Disorders: 2nd edition. Cephalalgia 2004; 24(Suppl. 1): 9-160.

12. Gallagher RM. Emergency treatment of intractable migraine. Headache 1986; 26: 74-75.

13. Fritsche G, Eberl A, Katsarava Z, et al. Drug-induced headache: Long-term follow-up of withdrawal therapy and persistence of drug misuse. Eur Neurol 2001; 45: 229-235.

14. Katsarava Z, Fritsche G, Muessig M, et al. Clinical features of withdrawal headache following overuse of triptans and other headache drugs. Neurology 2001; 57: 1694-1698.

15. Göbel H, Stolze H, Heinze A, et al. Easy therapeutical management of sumatriptan-induced daily headache. Neurology 1996; 47: 297-298.

16. Diener HC, Dodick DW, Goadsby PJ, et al. Utility of topiramate for the treatment of patients with chronic migraine in the presence or absence of acute medication overuse. Cephalalgia 2009; 29: 1021-1027. 